Macedonian Pharmaceutical Bulletin, 66 (Suppl 1) 131 - 132 (2020)

Online ISSN $1857-8969$

UDC: $615.454 .1 .014 .4: 617.7-085.281 .8$

DOI: 10.33320/maced.pharm.bull.2020.66.03.065

Short communication

\title{
Influence of sterilisation by irradiation on an antiviral eye ointment
}

\author{
Frosina Jovanovic*, Eleonora Trajanovska, Ana Atanasova, Emilija Arsovska Popovska, \\ Monika Stojanovska Pecova, Jelena Dimitrovska, Suzan Memed Sejfulah, \\ Packa Antovska, Sonja Ugarkovic
}

Research and Development, Alkaloid AD, Blvd. Aleksandar Makedonski 12, 1000 Skopje, North Macedonia

\section{Introduction}

Sterilisation by irradiation is becoming a commonly used method for sterilisation of pharmaceutical drug products that cannot be sterilised by other means such as sterilisation with dry heat, moist heat under pressure, gas, high intensity visible light or filtration. For semi-solid eye preparations filled in their final container sterilisation by irradiation can be considered as most suitable. (EMA, 2015; WHO, 2011)

In this study, we used an eye ointment in form of a suspension containing an antiviral agent as an active substance and white paraffin as an ointment base. Thermal sterilisation was not applicable considering the low melting point of the ointment base. Due to toxic residues problems or the high process costs, sterilisation with ethylene oxide was avoided. Sterilisation by irradiation was achieved by exposure of the product to gamma rays from cobalt $60\left({ }^{60} \mathrm{Co}\right)$ as an isotopic source, and to a beam of electrons energised by a suitable electron accelerator at the reference absorbed dose of $25 \mathrm{kGy}$. The procedure and precautions employed were such as to achieve sterility assurance level (SAL) equal to or less than $10^{-6}$ according to $\mathrm{Ph}$. Eur. 5.1.1 "Methods of preparation of sterile products" and ISO standard 11137.
The aim of this study was to evaluate the influence of gamma and e-beam irradiation at a dose of $25 \mathrm{kGy}$ when applied for the purpose of sterilisation of an antiviral eye ointment.

\section{Materials and methods}

An antiviral agent was used as an active substance and white paraffin was used as an ointment base. The manufacturing process and production equipment for the eye ointment included standard technological steps such as melting and mixing, dispersion of the active ingredient and homogenisation, cooling and filling. Sterilisation by irradiation was performed with an irradiator Gammatron 1500 with ${ }^{60}$ Co source type RSL 1800 and Rhodothron TT100-IBA-X, respectively for gamma and e-beam irradiation. The delivered doses were monitored using alanine dosimeters. Sterility tests of the samples were performed with $\mathrm{Ph}$. Eur. Method 2.6.1. "Sterility". The evaluation of the parameters, assay of active substance and the impurity profile, was done using a HPLC method. The particle size of the active substance in the eye ointment was monitored in accordance with Ph. Eur. monograph on semi-solid eye preparation (1163) using automated microscope Morphology G3. Viscosity analyses were performed using a

\footnotetext{
*fkralevska@alkaloid.com.mk
} 
Brookfield DV2T RV viscometer with a T-bar helipath spindle.

\section{Results and discussion}

The antiviral eye ointment was evaluated for its predetermined critical quality attributes (CQAs) including sterility and physico-chemical properties such as assay of active substance, related and degradation products, viscosity and particle size. The results from the tested product parameters were compared before and after performing the sterilisation by two types of irradiation.

Sterility is a fundamental issue in manufacture and use of eye products. Performed sterility tests for samples after sterilisation by irradiation showed that the sterility of the antiviral eye ointment was achieved when the product was irradiated by both gamma and e-beam irradiation with a dose of 25 kGy.

The main concern regarding sterilisation by irradiation, besides sterility assurance, is that the process should not cause any significant changes in the quality of the pharmaceuticals, specifically, alteration in the content of active ingredient or increase of impurities. All results for the parameter assay of antiviral active substance after sterilisation by gamma and e-beam irradiation were within the established acceptance criteria and no decrease was observed compared to the initial values obtained before sterilisation. The impurity profiles were similar before and after sterilisation by gamma and e-beam irradiation. From the data obtained for specified impurities, unspecified impurities and total impurities, it was concluded that all results were below the established acceptance criteria and no increase due to radiolysis was observed. Conclusively, it is not expected that the related and degradation products of the tested eye ointment could impose upon the safe use of the product.
The particle size of the active substance is a critical parameter for eye ointments containing suspended particles of the active substance. This parameter can affect the safety of the product, since if particle size is not within the acceptance criteria it can cause irritability in the eye. For this reason, the particle size of the active substance in the eye ointment was controlled by use of micronized grade and monitored during different stages of development and manufacture of the finished product. No change of particle size was observed between all tested samples before and after the sterilisation.

Rheological properties of ointments have a significant role in terms of processing and application. The viscosity analyses before and after sterilisation for all tested samples show no significant change of viscosity, therefore, the sterilization has no impact on the homogeneity of the product and on the application from the container.

\section{Conclusion}

Based on the obtained results for evaluated CQAs and taking into consideration the product characteristics, sterilisation by gamma and e-beam irradiation at a dose of $25 \mathrm{kGy}$ can be considered as suitable method for achieving sterility of the product. For both types of irradiation comparable effect on the quality of the antiviral eye ointment, regarding sterility and physico-chemical properties, was observed.

\section{References}

EMA/CHMP/CVMP/QWP/850374/2015, Guideline on sterilisation of the medicinal product, active substance, excipient and primary container.

European Pharmacopoeia 9th Edition (Ph. Eur. 9.0), Council of Europe, Strasbourg, France.

ISO standard 11137.

WHO Good manufacturing practices for sterile pharmaceutical products, 2011. WHO Technical Report Series, Annex 6. No. 961. 